\title{
Imaging the Tissue Response Around Brain-Implanted Microdevices
}

\author{
A.J. Woolley, ${ }^{*}$ H. Desai, ${ }^{*}$ and K.J. Otto*/** \\ * Department of Biological Sciences, Purdue University, 915 West State Street, West Lafayette, IN, \\ 47907-2054 \\ ** Weldon School of Biomedical Engineering, Purdue University, 206 South Martin Jischke Drive, \\ West Lafayette, IN, 47907-2032
}

Brain-implanted microdevices have the potential to interface with neurons in order to replace lost motor function or lost sensory function in human subjects [1]. However the usefulness of chronically implanted microdevices in the brain has been limited by an insulating response of the tissue to the device, as well as loss of nearby neurons [2]. Existing imaging methods to investigate this response require the removal of implanted microdevices prior to histological processing; this allows the tissue to be sliced without dragging or shattering the microdevices [3]. Unfortunately explanting the microdevices causes significant morphological damage to the interfacing tissue, often producing highly distorted explants holes due in part to the removal of tissue adhering to the device (FIG. 1a).

Using advanced labeling and imaging techniques, we have developed a method to examine histological sections that contain the intact microdevice and its tissue interface, allowing detailed imaging of the brain tissue response around implanted microdevices. The Device Capture Histology (DCHist) method has so far provided new insights into chronic inflammation and previously unreported tissue reorganization around in situ neural implants. Looking at superficial rat motor cortex, microglia, the immune cells of the brain, have been revealed to be highly present around the intact device (FIG. 1b-c). Oligodendrocytes, cells which insulate neuron axons in the brain, appear to be disrupted by the microdevice but are still present along the interface (FIG. 1d). However, astrocytes (FIG. 1e), cells that primarily support healthy neural environment, are not prominent at the intact device/tissue interface, but are present in an atypically elongated form just outside of the predominantly microglial peri-electrode space.

Because the DCHist method produces thicker ( $>100 \mathrm{um})$ tissue sections, we have also used 2-photon microscopy as well as 'tissue clearing' techniques to image further into tissue specimens. These results have allowed closer investigation of the intact tissue interface surrounding brainimplanted microdevices in thick histological sections.

Finally, we have initiated in vivo brain tissue imaging of the tissue response to implanted device. These data, taken through cranial windows installed over implanted devices, are allowing characterization of the developing brain response to implanted microdevices over time.

\section{References}

[1] K.J. Otto et al., Hearing Research 210 (2005) 112

[2] R. Biran et al., Experimental Neurology 195 (2005) 115

[3] J.N. Turner et al. Experimental Neurology 156 (1999) 33 

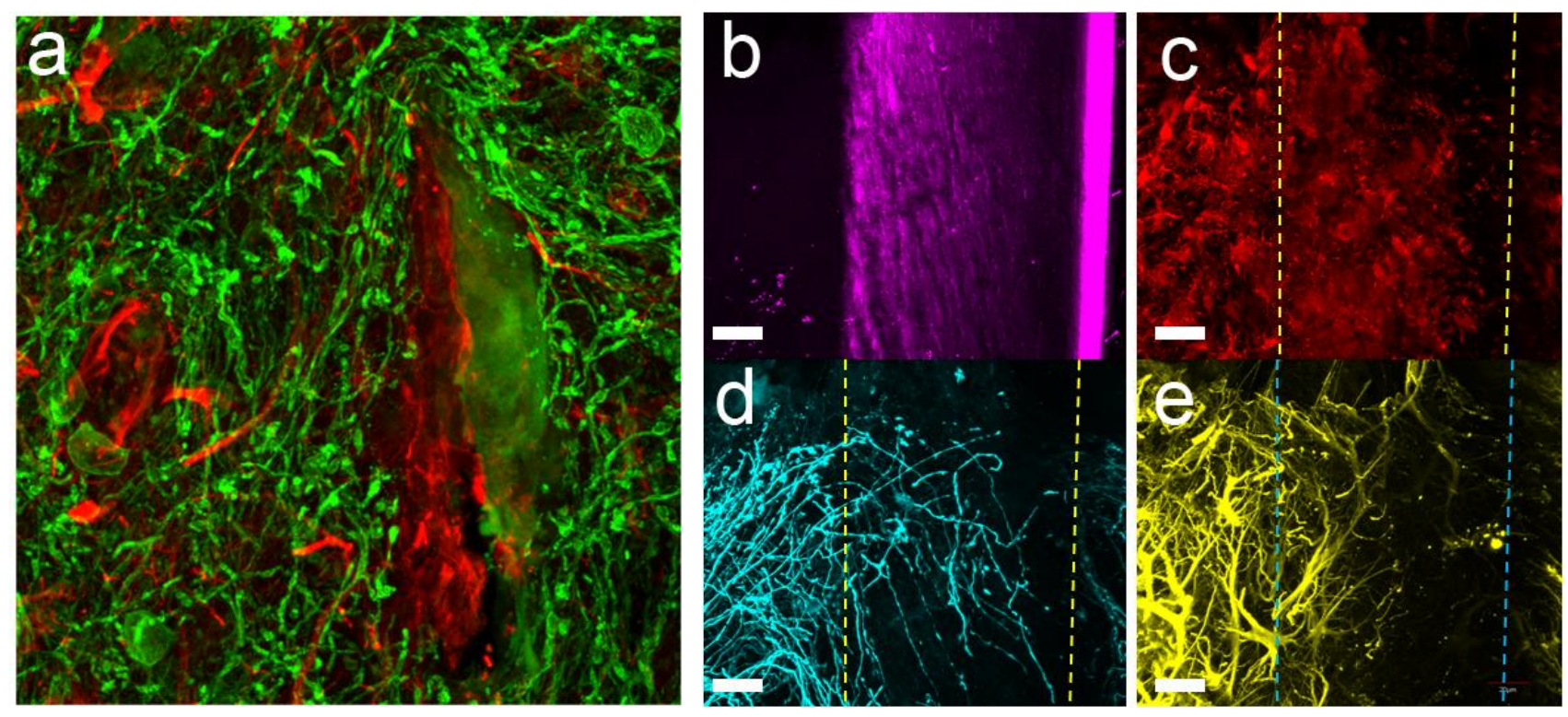

FIG. 1. Histology from the site of explanted and captured microdevices in brain tissue. Part (a) shows a slit remaining in tissue from which a 2 week implanted microdevice was extracted; the former device/tissue interface has closed-up and only a complex response remains; green indicate an oligodendrocyte-specific antigen while red are astrocytes and microglia antigens. Part (b) shows a captured microdevice (magenta) within a brain slice collect within a slice of superficial rat motor cortex, imaged by capturing reflectance off of the device surface. Microglia (c, red), oligodendrocytes (d, cyan), and astrocytes (e, yellow) and seen interacting with this surface, with micoglia and some oligodendrocytes up against the device surface, while astrocytes are primarily relegated to a longer distance away from the silicon device. Images collected with a laser scanning confocal microsope, with image (a) being a $\sim 10 \mu \mathrm{m}$ thick highest-intensity projection and (b-e) a $\sim 60 \mu \mathrm{m}$ thick highest-intensity projections. Scale bars: $10 \mu \mathrm{m}$ in (a), $20 \mu \mathrm{m}$ in (b-e). 